\title{
Studies on the reactions of thiocarbonyl s-methanides with hetaryl thioketones
}

\author{
Mlostoń, Grzegorz ; Pipiak, Paulina ; Linden, Anthony ; Heimgartner, Heinz
}

\begin{abstract}
Dihetaryl thioketones react with thiocarbonyl ylides to give 1,3-dithiolanes in high yields. No competitive side reactions of the thiocarbonyl ylides were observed, evidencing the 'superdipolarophilic' character of this less-known group of thioketones. Depending on the type of substituents present in both the thiocarbonyl ylide and the thioketone, formal [3+2] cycloadditions occur with complete regioselectivity or with formation of a mixture of both regioisomers. Regioselective formation of the sterically more crowded 1,3-dithiolanes is explained via a mechanism involving stabilized 1,5-biradicals. In systems with less-efficient radical stabilization, e.g., in the case of adamantanethione S-methanide, substantial violation of the regioselectivity was observed as a result of steric hindrance.
\end{abstract}

DOI: https://doi.org/10.1002/hlca.201500057

Posted at the Zurich Open Repository and Archive, University of Zurich

ZORA URL: https://doi.org/10.5167/uzh-110375

Journal Article

Accepted Version

Originally published at:

Mlostoń, Grzegorz; Pipiak, Paulina; Linden, Anthony; Heimgartner, Heinz (2015). Studies on the reactions of thiocarbonyl s-methanides with hetaryl thioketones. Helvetica Chimica Acta, 98(4):462-473.

DOI: https://doi.org/10.1002/hlca.201500057 


\title{
Studies on the Reactions of Thiocarbonyl $S$-Methanides with Hetaryl
}

\section{Thioketones}

\author{
by Grzegorz Mlostoń*a ${ }^{\mathbf{a}}$ ), Paulina Pipiak ${ }^{\mathrm{a}}{ }^{1}$ ), Anthony Linden ${ }^{\mathrm{b}}$ ), and Heinz \\ Heimgartner*b)
}

a) University of Łódź, Department of Organic and Applied Chemistry, Tamka 12, PL91-403 Łódź (phone: +48 42 6355761; fax: +48 42 6655162; e-mail: gmloston@uni.lodz.pl)

b) University of Zürich, Department of Chemistry, Winterthurerstrasse 190, CH-8057 Zürich (phone:+41 44 6354282; fax: +41 44 6356812; e-mail: heinz.heimgartner@chem.uzh.ch)

$\left.{ }^{1}\right)$ Part of the planned PhD thesis of $P . P$. , University of Łódź 
Bishetaryl thioketones react with thiocarbonyl ylides to give 1,3-dithiolanes in high yields. No competitive side reactions of the thiocarbonyl ylides are observed, evidencing the 'superdipolarophilic' character of this little-known group of thioketones. Depending on the type of substituents present in both the thiocarbonyl ylide and the thioketone, formal [3+2] cycloadditions occur with complete regioselectivity or with formation of a mixture of both regioisomers. Regioselective formation of the sterically more crowded 1,3-dithiolanes is explained via a mechanism involving stabilized 1,5biradicals. In systems with less efficient radical stabilization, e.g., in the case of adamantanethione $S$-methanide, substantial violation of the regioselectivity is observed as a result of steric hindrance. 
1. Introduction. - Thiocarbonyl ylides belong to the class of the so-called Scentered 1,3-dipoles, and they are widely applied for the preparation of S-heterocycles with diverse sizes of the formed ring [1]. The reactive thiocarbonyl S-methanides, generated via thermal $\mathrm{N}_{2}$-elimination from 2,5-dihydro-1,3,4-thiadiazoles, easily react with diverse dipolarophiles, and special attention is focused on their reactions with 'superdipolarophilic' thioketones [2]. These reactions leading to 1,3-dithiolane derivatives (Schönberg reaction [3]) are of interest not only as a method for the preparation of these products, but also for studies on organic reaction mechanisms. Important features of these reactions are the regioselectivity and the nature of the postulated intermediates. Whereas S-methanides of cycloaliphatic thioketones, e.g., adamantanethione S-methanide (1a), react with adamantanethione (2a) to give the sterically less hindered 2,2,4,4-tetrasubstituted 1,3-dithiolane of type 3a, its reaction with thiofluorenone (2b) gave a ca. 1:3 mixture of the regioisomeric 1,3-dithiolanes $\mathbf{3 b}$ and $\mathbf{4 b}$ in favor of the sterically more crowded isomer [4] (Scheme 1). On the other hand, S-methanides derived from aromatic thioketones, e.g., thiobenzophenone Smethanide (1b), react with thiobenzophenone (2c) to give the 4,4,5,5-tetrasubstituted 1,3-dithiolane of type $\mathbf{4 c}$ as the sole product [3b].

\section{Scheme 1}

In a recent publication, we described the synthesis and selected reactions of aryl/hetaryl and bishetaryl thioketones [5]. Unexpectedly, the experiments with diazomethane demonstrated that the presence of a hetaryl substituent such as thiophen2-yl, selenophen-2-yl, or furan-2-yl results in a spontaneous evolution of $\mathrm{N}_{2}$ even at $60^{\circ}$. In contrast to thiobenzophenone, the precursor of the corresponding S-methanides 
of type 1, i.e., 2,5-dihydro-2,2-diaryl-1,3,4-thiadiazoles 5, could not be prepared in solution. Instead, a reactive intermediate trapped the starting thioketone $\mathbf{2}$ and yielded the sterically more crowded 1,3-dithiolane in a regioselective manner. However, in the case of phenyl (selenophen-2-yl) thioketone (2d), the formation of a second product, a novel macrocyclic dimer of the thiocarbonyl S-methanide was observed [6]. Based on these results, we proposed that the intermediate 'thiocarbonyl ylide' displays a biradical character. Thus, the formation of the 12-membered cyclodimer can be considered as experimental evidence for the appearance of a delocalized biradical. An earlier computational study suggested the participation of a 1,5-diradical in the formation of 1,3-dithiolane derivatives via formal [3+2] cycloaddition of thioketones with thiocarbonyl S-methanides [7].

The goal of the present study was the investigation of the formation of 1,3dithiolanes via formal [3+2] cycloaddition of thiocarbonyl S-methanides and aryl/hetaryl and bishetaryl thioketones. Of special interest is the regioselectivity of the ring formation.

2. Results and Discussion. - The thermal decomposition of 2,2-disubstituted 2,5-dihydro-1,3,4-thiadiazoles $\mathbf{5}$ is considered as a straightforward method for the in situ generation of reactive thiocarbonyl S-methanides of type 1 [1a][8]. The stability of these precursors depends strongly on the type of substitutents, and the presence of bulky cycloaliphatic groups allows them to be prepared as crystalline, shelf-stable compounds. On the other hand, 2,2-diaryl-substituted 2,5-dihydro-1,3,4-thiadiazoles 5 can be prepared only at low temperature and have to be used as thiocarbonyl S-methanide precursors at $-40^{\circ}$ without isolation. 
Based on the typical procedure for the generation of diaryl-substituted thiocarbonyl S-methanides of type $\mathbf{1 b}$, solutions of thiobenzophenone (2c) or thiofluorenone (2b) in THF were treated with $\mathrm{CH}_{2} \mathrm{~N}_{2}$ at $-60^{\circ}$. Equimolar amounts of hetaryl/phenyl (2d, 2e) or bishetaryl thioketones (2f, $\mathbf{2 g})$ were added to the colorless solutions and the mixtures were warmed to $-40^{\circ}$. After complete evolution of $\mathrm{N}_{2}$, the

crude products were analyzed by ${ }^{1} \mathrm{H}-\mathrm{NMR}$ spectroscopy with a weighed amount of 1,1,2,2-tetrachloroethane as a standard. In all reactions, only one product was detected in good yields and identified as the sterically crowded 4,4,5,5-tetrasubstituted 1,3dithiolanes 4 (Scheme 2). The proposed structures of the products were elucidated from the characteristic absorption of $\mathrm{H}_{2} \mathrm{C}(2)$ in the ${ }^{13} \mathrm{C}-\mathrm{NMR}$ spectra at 30.5-32.5 ppm [3].

\section{Scheme 2}

In the second series, adamantanethione S-methanide (1a) was generated from its precursor $5 \mathbf{a}$ at $45^{\circ}$ in THF in the presence of equimolar amounts of thioketones $\mathbf{2}$. In all cases, the formation of mixtures of regioisomeric 1,3-dithiolanes was shown by ${ }^{1} \mathrm{H}$ NMR spectroscopy (weighed standard). In analogy to earlier reported products obtained from $1 \mathbf{a}$ and aromatic thioketones $\mathbf{2 b}$ and $\mathbf{2 c}$ (Scheme 1), the major products were identified as the sterically more crowded 4,4,5,5-tetrasubstituted 1,3-dithiolanes of type 4 (Scheme 3). The ratios of the isomers 3 and $\mathbf{4}$ established by NMR spectroscopy were between $c a$. 1:3 and 1:4, and in the case of the mixtures of $\mathbf{3 e} / \mathbf{4} \mathbf{k}$ and $\mathbf{3 h} / \mathbf{4 n}$ the major products $4 \mathbf{k}$ and $\mathbf{4 n}$, respectively, were isolated as pure crystalline compounds and fully characterized. 
Reactions with the 2,2,4,4-tetramethyl-3-oxocyclobutanethione S-methanide (1c) with hetaryl phenyl thioketones $\mathbf{2} \mathbf{d}$ and $\mathbf{2 e}$ as well as with the bishetaryl thioketone $\mathbf{2 h}$ led also to mixtures of regioisomeric 1,3-dithiolanes $\mathbf{3}$ and $\mathbf{4}$ with the sterically more congested product of type $\mathbf{4}$ as the major component (Scheme 4). In comparison with the series of 1,3-dithiolanes obtained in the case of 1a (Scheme 3), the ratio of isomers increased in favor of the more crowded isomers of type 4. However, the analogous reactions with bis(thiophen-2-yl) thioketone (2f) and bis(selenophen-2-yl) thioketone (2g) led to the sterically more crowded 1,3-dithiolanes $\mathbf{4 r}$ and $\mathbf{4 s}$, respectively, as the sole products.

\section{Scheme 4}

Finally, the structure of compound $\mathbf{4 r}$, which had been deduced from the NMR data, was unambiguously confirmed by X-ray crystallography (Figure). Although the molecule is achiral, the compound has crystallized in a chiral space group and the absolute structure has been determined by the diffraction experiment. There are two symmetry-independent molecules in the asymmetric unit. Both molecules show disorder of the thiophene rings due to $180^{\circ}$ rotation of each ring around its parent $\mathrm{C}-\mathrm{C}$ bond. The 5-membered dithiolane ring in each molecule has a half-chair conformation twisted on the $\mathrm{C}-\mathrm{C}$ bond.

Figure. ORTEP plot [9] of the molecular structure of one of the two symmetryindependent molecules of $\mathbf{4 r}$ (with $50 \%$ probability ellipsoids; arbitrary numbering of the atoms; only the major conformations of the disordered thiophene rings are shown). 
It is worth mentioning that the thiocarbonyl ylides 1a-1d reacted with hetaryl thioketones 2 without competitive formation of 1,4-dithianes or thiiranes (see [1a][8]). These results confirm that hetaryl thioketones belong to the group of 'superdipolarophiles' in reactions with thiocarbonyl S-methanides [2]. According to Huisgen's reactivity scale, the most reactive thioketone is thiofluorenone $(\mathbf{2 b})$, followed by thiobenzophenone (2c). For comparison reasons, the competition experiments of thiobenzophenone S-methanide (1) with equimolar amounts of $\mathbf{2 b}$ and bis(thiophen-2yl) thioketone (2f), as well as with $\mathbf{2 c}$ and $\mathbf{2 f}$, were performed in THF at $c a$. $-40^{\circ}$. The obtained products were analyzed by ${ }^{1} \mathrm{H}-\mathrm{NMR}$ spectroscopy. In the first case, the only product formed was the sterically crowded 1,3-dithiolane $\mathbf{4 h}$. In the second experiment, however, the ratio of $\mathbf{4 c}$ (Scheme 1) to $\mathbf{4 f}$ was determined to be $c a$. 5:4. These results demonstrate that the symmetrical hetaryl thioketone $\mathbf{2 f}$ is less reactive than thiofluorenone (2b) but almost as reactive as thiobenzophenone (2c).

3. Conclusions. - The present study showed that hetaryl thioketones extend the group of 'superdipolarophiles' in reactions with thiocarbonyl S-methanides 1 . The observed regioselectivity of the 1,3-dithiolane formation suggests that the formal $[3+2]$ cycloaddition occurs, most likely, via biradical intermediates. The latter mechanism leads to complete regioselectivity only when the substituents stabilize the biradical structure, e.g., via delocalization in tetraaryl-substituted systems ( $c f$. [6]). In such systems, the intermediate 1,5-diradical $\mathbf{6 a}$ is the precursor of the sterically crowded 4,4,5,5-tetrasubstituted 1,3-dithiolane, such as 4d-4h [7] (Scheme 2). The same regioisomers were only obtained as sole products in the reaction of 2,2,4,4-tetramethyl3-oxocyclobutanethione S-methanide (1c) with bishetaryl thioketones $\mathbf{2 f}$ and $\mathbf{2 g}$, which 
possess $\mathrm{S}$ or Se atoms in both heterocycles. In these cases, the stabilization of the 1,5biradical intermediate $\mathbf{6 b}$ results from delocalization within the hetaryl rings and, likely, from an additional stabilizing effect across the cyclobutanone ring [10]. The presence of $\mathrm{Se}$ or $\mathrm{S}$ atoms in the five-membered heteroaromatic rings is essential for the exclusive formation of an intermediate of type $\mathbf{6 b}$ (see also [6]). Both heteroatoms are known to stabilize radicals in the $\alpha$-position.

\section{Formulae 6a and $\mathbf{6 b}$}

In the presented cases, electronic effects are decisive, but in the reactions with adamantanethione S-methanide (1a), no radical stabilization is possible at the adamantane-substituted terminus of the intermediate. For that reason, the influence of steric factors is of increasing importance and the formation of the sterically less crowded 1,3-dithiolanes as minor products is also observed in all cases.

In summary, the results discussed in this publication, additionally supported by computational studies [7], evidence that in the case of S-centered thiocarbonyl ylides some of the formal [2+3] cycloadditions occur stepwise via biradical intermediates. This conclusion supports the concept of the radical character of '1,3-dipolar cycloaddition reactions' formulated by Firestone [11], at least for some systems with the required structural features.

The authors thank the National Science Center (PL-Cracow) for financial support within the project Maestro (Grant Maestro-3; Dec-2012/06/A/ST5/00219). 


\section{Experimental Part}

1. General. M.p.: MEL-TEMP. II (Aldrich); uncorrected. Column chromatography (CC): silica gel (70-230 mesh, Merck). IR Spectra: NEXUS FT-IR instrument; in $\mathrm{KBr}$; absorptions in $\mathrm{cm}^{-1}$. ${ }^{1} \mathrm{H}-\mathrm{NMR}$ and ${ }^{13} \mathrm{C}-\mathrm{NMR}$ Spectra: BRUKER AVANCE III instrument $\left({ }^{1} \mathrm{H}\right.$ at 600 and ${ }^{13} \mathrm{C}$ at $\left.150 \mathrm{MHz}\right)$ using the solvent signal as reference; in $\mathrm{CDCl}_{3}$; chemical shifts $(\delta)$ in ppm; coupling constants $J$ in Hz. ESI-MS: Varian 500 MS LC Ion Trap spectrometer. Elemental analyses were performed in the Laboratory of the Faculty of Chemistry, University of Łódź.

2. Starting Materials. 1,1,3,3-Tetramethyl-5-thia-7,8-diazaspiro[3.4]oct-7-en-2one (5d) [12] and 2,5-Dihydrospiro[1,3,4-thiadiazole-2,2'-tricyclo[3.3.1.1 ${ }^{3,7}$ ]decane] (5a) [13] were prepared by known methods according to the literature protocols. Thiobenzophenone (2b), fluorene-9-thione (2c), the nonsymmetrical heteroaromatic thioketones phenyl(selenophen-2-yl)methanethione (2d), phenyl(selenophen-2$y l)$ methanethione (2e), and (furan-2-yl) (thiophen-2-yl)methanethione (2h), as well as the symmetrical heteroaromatic thioketones bis(thiophen-2-yl)methanethione (2g) and bis(selenophen-2-yl)methanethione (2h) were obtained from the corresponding ketones using the known thionation procedure with Lawsson's reagent [5]. Other reagents used in the present study were commercially available.

3. Reactions of Hetaryl Phenyl Thioketones $\mathbf{2 d - 2 e ~ o r ~ B i s h e t a r y l ~ T h i o k e t o n e s ~} \mathbf{2 f -}$ $2 \mathrm{~g}$ with 2,5-Dihydro-2,2-diphenyl-1,3,4-thiadiazole (5b). - General Procedure. Thiobenzophenone (2b, $1 \mathrm{mmol})$ dissolved in $2 \mathrm{ml}$ of THF was cooled to $-70^{\circ}$ and treated with small portions of ethereal $\mathrm{CH}_{2} \mathrm{~N}_{2}$ soln. until the dark-blue color disappeared. A soln. of the corresponding thioketone $\mathbf{2 d}-\mathbf{g}(1 \mathrm{mmol})$ in $2 \mathrm{ml}$ of $\mathrm{THF}$ was added at $-70^{\circ}$ and the reaction mixture was kept in a cold bath $\left(-45^{\circ}\right.$ to $-40^{\circ}$, acetone/dry ice) for $2.5 \mathrm{~h}$. Then, the mixture was allowed to warm slowly to r.t. During 
the reaction, a colorless precipitate was formed. The mixture was kept at r.t. for $c a .30$ min. Then, the solvent was evaporated; the crude product was purified by crystallization or by treatment with small portions of hexane.

3.1. 4,5,5-Triphenyl-4-(thiophen-2-yl)-1,3-dithiolane (4d). Reaction with 2d; the crude product was purified by crystallization from $\mathrm{CH}_{2} \mathrm{Cl}_{2} /$ hexane. Yield: $320 \mathrm{mg}$ (77\%). Colorless crystals. M.p. 175.2-176.5 $\left(\mathrm{CH}_{2} \mathrm{Cl}_{2} /\right.$ hexane). IR (KBr): 3050w, 1597w, 1488m, 1440m, 1233m, 1033w, 747m, 722m, 708s. ${ }^{1} \mathrm{H}-\mathrm{NMR}\left(\mathrm{CDCl}_{3}\right): 3.01$, $3.89\left(A B, J_{\mathrm{AB}}=9.6, \mathrm{CH}_{2}\right) ; 6.76-6.77(m, 1$ arom. $\mathrm{H}) ; 6.85(d, J=3.6,1$ arom. $\mathrm{H}) ; 7.10$ 7.20 (m, 10 arom. H); 7.39-7.43 (m, 4 arom. $\mathrm{H}) ; 7.50$ (d, $J=8.4,2$ arom. $\mathrm{H}) .{ }^{13} \mathrm{C}-\mathrm{NMR}$ $\left(\mathrm{CDCl}_{3}\right): 30.5\left(\mathrm{H}_{2} \mathrm{C}(2)\right) ; 74.5,78.3(\mathrm{C}(4), \mathrm{C}(5)) ; 125.1,125.6,126.3,126.4,126.6$, 126.7, 126.8, 127.0, 130.0, 130.6, 131.4, 131.8 (18 arom. CH); 142.3, 143.0 (br., 4 arom. C). HR-ESI-MS: 416.072719 (calc. for $\mathrm{C}_{25} \mathrm{H}_{20} \mathrm{~S}_{3} 416.073100, M^{+}$).

3.2. 4,5,5-Triphenyl-4-(selenophen-2-yl)-1,3-dithiolane (4e). Reaction with 2e; the crude product was purified by crystallization from $\mathrm{CH}_{2} \mathrm{Cl}_{2} /$ hexane. Yield: $320 \mathrm{mg}$ (70\%). Colorless crystals. M.p. 177.4-178.8 $\left(\mathrm{CH}_{2} \mathrm{Cl}_{2} /\right.$ hexane). IR (KBr): $3049 w$, $1597 w, 1489 m, 1439 m, 1232 m, 1186 w, 1083 w, 1034 w, 746 m, 716 s, 696 s .{ }^{1} \mathrm{H}-\mathrm{NMR}$ $\left(\mathrm{CDCl}_{3}\right): 3.81,3.90\left(A B, J_{\mathrm{AB}}=9.6, \mathrm{CH}_{2}\right) ; 7.00-7.01(m, 2$ arom. $\mathrm{H}) ; 7.07-7.19(m, 9$ arom. H); 7.37 ( $d, J=7.2,2$ arom. $\mathrm{H}) ; 7.46-7.50$ (m, 4 arom. $\mathrm{H}) ; 7.89(d d, J=4.8,1.8$, 1 arom. H). ${ }^{13} \mathrm{C}-\mathrm{NMR}\left(\mathrm{CDCl}_{3}\right): 30.6\left(\mathrm{H}_{2} \mathrm{C}(2)\right) ; 76.4,78.2(\mathrm{C}(4), \mathrm{C}(5)) ; 126.2,126.6$, 126.7, 126.7, 126.9, 127.1, 127.9, 130.6, 131.6, 131.8, 131.9 (18 arom. CH); 142.7, 142.8 (br., 4 arom. C). Anal. calc. for $\mathrm{C}_{25} \mathrm{H}_{20} \mathrm{~S}_{2} \mathrm{Se}$ (463.53): C 64.78, H 4.35, S 13.83; found: C 64.55, H 4.38, S 13.75.

3.3. 5,5-Diphenyl-4,4-bis(thiophen-2-yl)-1,3-dithiolane (4f). Reaction with 2f; the crude product was purified by repeated treatment with small portions of hexane. Yield: 320 mg (76\%). Colorless crystals. M.p. 192.7-193.4. IR (KBr): 3095w, 3068w, 
1595w, 1488m, 1440m, 1238m, 1086w, 849w, 747s, 734m, 707s. ${ }^{1} \mathrm{H}-\mathrm{NMR}\left(\mathrm{CDCl}_{3}\right)$ : $3.94\left(s, \mathrm{CH}_{2}\right) ; 6.80(d d, J=5.4,3.6,2$ arom. $\mathrm{H}) ; 7.03(d d, J=3.6,1.2,2$ arom. $\mathrm{H}) ; 7.10$ 7.18 (m, 8 arom. H); 7.40-7.41 (m, 4 arom. H). ${ }^{13} \mathrm{C}-\mathrm{NMR}\left(\mathrm{CDCl}_{3}\right): 31.4\left(\mathrm{H}_{2} \mathrm{C}(2)\right) ; 71.4$, 79.1 (C(4), C(5)); 125.6, 125.7, 126.5, 126.9, 129.1, 131.4 (16 arom. CH); 142.2, 147.9 (4 arom. C). ESI-MS (MeOH): $461\left(55,[M+\mathrm{K}]^{+}\right), 445\left(45,[M+\mathrm{Na}]^{+}\right), 423(100$, $\left.[M+\mathrm{H}]^{+}\right)$. Anal. calc. for $\mathrm{C}_{23} \mathrm{H}_{18} \mathrm{~S}_{4}$ (422.64): C 65.36, H 4.29, S 30.35; found: C 64.81, H 4.39, S 30.32.

3.4. 5,5-Diphenyl-4,4-bis(selenophen-2-yl)-1,3-dithiolane (4g). Reaction with $\mathbf{2 g}$; the crude product was purified by repeated treatment with small portions of hexane. Yield: $310 \mathrm{mg}$ (58\%). Colorless crystals. M.p. 184.8-185.3․ IR (KBr): 3096w, 3050w, $1624 w, 1487 w, 1439 m, 1227 m, 1033 w, 729 m, 697 s .{ }^{1} \mathrm{H}-\mathrm{NMR}\left(\mathrm{CDCl}_{3}\right): 3.99\left(s, \mathrm{CH}_{2}\right)$; 7.08-7.09 (m, 2 arom. H); 7.13-7.19 (m, 6 arom. H); 7.30 (dd, J=4.2, 1.2, 2 arom. H ); 7.45-7.46 (m, 4 arom. H); 7.89 (dd, $J=5.4,1.2,2$ arom. H). ${ }^{13} \mathrm{C}-\mathrm{NMR}\left(\mathrm{CDCl}_{3}\right): 31.6$ $\left(\mathrm{H}_{2} \mathrm{C}(2)\right) ; 75.2,78.9(\mathrm{C}(4), \mathrm{C}(5)) ; 126.5,127.0,128.3,130.9,131.5,132.1$ (16 arom. $\mathrm{CH}$ ); 142.4, 145.0 (br., 4 arom. C). Anal. calc. for $\mathrm{C}_{23} \mathrm{H}_{18} \mathrm{~S}_{2} \mathrm{Se}_{2}$ (516.44): C 53.49, H 3.51, S 12.42; found: C 53.58, H 3.65, S 12.63.

4. Reaction of 2,5-Dihydrospiro[1,3,4-thiadiazole-2,9'-[9H]fluorene] (5c) with Bis(thiophen-2-yl)methanethione (2f). A soln. of fluorene-9-thione (2c, $1 \mathrm{mmol})$ in $2 \mathrm{ml}$ of THF was cooled to $-70^{\circ}$ and treated with small portions of a soln. of $\mathrm{CH}_{2} \mathrm{~N}_{2}$ in $\mathrm{Et}_{2} \mathrm{O}$, until the green soln. became yellow. Then, a soln. of $\mathbf{2 f}(1 \mathrm{mmol})$ in $2 \mathrm{ml}$ of THF was added at $-70^{\circ}$ and the mixture was kept in a cold bath $\left(-45^{\circ}\right.$ to $-40^{\circ}$, acetone/dry ice $)$ for $1 \mathrm{~h}$. Then, the mixture was allowed to warm slowly to r.t., whereby a colorless precipitate was formed. The mixture was kept at r.t. for $c a .30$ min, then the solvent was evaporated, and the residue was crystallized from $\mathrm{CH}_{2} \mathrm{Cl}_{2} /$ hexane. 
5,5-Diphenylspiro[1,3-dithiolane-4,9'-[9H]fluorene] (4h). Yield: $295 \mathrm{mg}$ (70\%). Colorless crystals. M.p. 216.4-218.0 ${ }^{\circ}\left(\mathrm{CH}_{2} \mathrm{Cl}_{2} /\right.$ hexane). IR (KBr): 3066w, $2924 w, 1624 w, 1446 m, 1427 w, 1227 m, 1239 m, 1048 w, 1034 w, 851 w, 789 s, 745 s, 736 s$, $719 s, 706 s .{ }^{1} \mathrm{H}-\mathrm{NMR}\left(\mathrm{CDCl}_{3}\right): 4.50\left(s, \mathrm{CH}_{2}\right) ; 6.61(d d, J=3.6,1.2,2$ arom. $\mathrm{H}) ; 6.74$ $(d d, J=5.4,3.6,2$ arom. H); 7.07-7.13 (m, 6 arom. H); 7.32-7.35 (m, 2 arom. H); 7.69 $\left(d,{ }^{2} J_{\mathrm{H}, \mathrm{H}}=7.2,2\right.$ arom. H). ${ }^{13} \mathrm{C}-\mathrm{NMR}\left(\mathrm{CDCl}_{3}\right): 32.5\left(\mathrm{H}_{2} \mathrm{C}(2)\right) ; 70.3,75.5(\mathrm{C}(4), \mathrm{C}(5))$; $119.8,125.3,125.7,125.9,127.5,127.8,128.6$ (14 arom. CH); 140.2, 144.1, 147.9 (6 arom. C). ESI-MS (MeOH): $443\left(100,[M+\mathrm{Na}]^{+}\right)$.

5. Reactions of Hetaryl Phenyl Thioketones $\mathbf{2 d - 2 e ~ o r ~ B i s h e t a r y l ~ T h i o k e t o n e s ~} \mathbf{2 f -}$ 2h with 2,5-Dihydrospiro[1,3,4-thiadiazole-2,2'-tricyclo[3.3.1.1 $\left.1^{3,7}\right]$ decane] (5a). General Procedure. The thiadiazoline $\mathbf{5 a}(1.1 \mathrm{mmol})$ and a corresponding thioketone 2 (1.05 mmol) were dissolved in freshly distilled THF $(2.5 \mathrm{ml})$. The mixture was heated in an oil bath $\left(45-50^{\circ}\right)$ until the intense color of the thioketone vanished; the gas burette combined with the flask indicated the evolution of stoichiometric amounts of $\mathrm{N}_{2}$. After removal of the solvent under vacuum, the residue was subjected to ${ }^{1} \mathrm{H}-\mathrm{NMR}$ analysis in $\mathrm{CDCl}_{3}$ soln. with a weighed amount of 1,1,2,2-tetrachloroethane as standard. Crude products were purified by $\mathrm{CC}\left(\mathrm{CH}_{2} \mathrm{Cl}_{2} /\right.$ petroleum ether 4:6). In all cases, formation of mixtures of regioisomeric 1,3-dithiolanes $\mathbf{3}$ and $\mathbf{4}$ was observed.

5.1. Reaction with 2d: 5-Phenyl-5-(thiophen-2-yl)spiro[1,3-dithiolane-4,2'tricyclo[3.3.1.1 $1^{3,7}$ decane] (4i; major) and 4-Phenyl-4-(thiophen-2-yl)spiro[1,3dithiolane-2,2'-tricyclo[3.3.1.1 $1^{3,7}$ ]decane] (3d; minor); crude product ratio: 75:25. Reaction time: $8 \mathrm{~h}$. Isolated as a mixture of isomers. Yield: $332 \mathrm{mg}(82 \%)$. Yellow crystals. M.p.139.8-141.0. IR (KBr): 2905s, 2855s, 1443m, 1233w, 1220w, 1099w, $743 w, 708 w .{ }^{1} \mathrm{H}-\mathrm{NMR}\left(\mathrm{CDCl}_{3}\right): 1.19-3.04(m, 28 \mathrm{H}) ; 3.50,3.56\left(A B, J_{\mathrm{AB}}=9.0, \mathrm{CH}_{2}\right.$ of 4i); 3.83, $3.94\left(A B, J=12.6, \mathrm{CH}_{2}\right.$ of $\left.3 \mathbf{d d}\right) ; 6.78-7.94\left(m, 16\right.$ arom. H). ${ }^{13} \mathrm{C}-\mathrm{NMR}$ 
$\left(\mathrm{CDCl}_{3}\right): 26.3,26.4,26.6,26.8,36.4,36.8,42.0,42.1\left(8 \mathrm{CH}_{(\mathrm{ad})}\right) ; 27.2\left(\mathrm{H}_{2} \mathrm{C}(2)\right.$ of $\left.4 \mathbf{i}\right)$; $33.2,33.6,36.5,36.5,36.6,36.9,37.6,38.2,38.9,40.5\left(10 \mathrm{CH}_{2(\mathrm{ad})}\right) ; 50.2\left(\mathrm{H}_{2} \mathrm{C}(5)\right.$ of 3d); 70.5, 75.4, 77.6, 78.5 (2 C(4), 2 C(5)); 124.8, 125.0, 126.0, 126.1, 126.6, 127.2, 127.4, 127.9, 128.1, 128.8, 130.8 (16 arom. CH); 140.8, 148.5 (2 arom. C of 4i); 144.0, 151.2 (2 arom. C of 3d). Anal. calc. for $\mathrm{C}_{22} \mathrm{H}_{24} \mathrm{~S}_{3}$ (384.63): C 68.70 H 6.29 S 25.01; found: C 68.37 H 6.00 S 25.01 .

5.2. Reaction with 2e: 5-Phenyl-5-(selenophen-2-yl)spiro[1,3-dithiolane-4,2'tricyclo[3.3.1.1 $\left.{ }^{3,7}\right]$ decane] (4k; major) and 4-Phenyl-4-(selenophen-2-yl)spiro[1,3dithiolane-2,2'-tricyclo[3.3.1.1.7, $]$ decane] (3e; minor); crude product ratio: 78:22. Reaction time: $7 \mathrm{~h}$. After chromatography, $\mathbf{4 k}$ and $\mathbf{3 e}$ were isolated as a mixture of regioisomers. Yield: $358 \mathrm{mg}(80 \%)$. After repeated crystallization from hexane, the major product 4k was isolated in pure form. Colorless crystals. M.p. 152.1-153.0 (hexane). IR (KBr): 2903s, 2858m, 1441m, 1231m, 1221m, 709s, 695m, 684s. ${ }^{1} \mathrm{H}-\mathrm{NMR}$ $\left(\mathrm{CDCl}_{3}\right)$ : 1.04-3.05 $(m, 14 \mathrm{H}) ; 3.54,3.60\left(A B, J_{\mathrm{AB}}=8.4, \mathrm{CH}_{2}\right)$; 7.17-7.96 ( $m, 4$ arom. $\mathrm{H}) ; 7.59\left(d,{ }^{2} J_{\mathrm{H}, \mathrm{H}}=3.6,1\right.$ arom. $\left.\mathrm{H}\right) ; 7.83\left(d,{ }^{2} J_{\mathrm{H}, \mathrm{H}}=6.6,1\right.$ arom. $\left.\mathrm{H}\right) ; 7.96$ (br. $s, 2$ arom. H). ${ }^{13} \mathrm{C}-\mathrm{NMR}\left(\mathrm{CDCl}_{3}\right): 26.7,26.9,36.4,37.1\left(4 \mathrm{CH}_{(\mathrm{ad})}\right) ; 27.5\left(\mathrm{H}_{2} \mathrm{C}(2)\right) ; 33.2,33.3,38.0$, 39.0, $40.8\left(5 \mathrm{CH}_{2(\mathrm{ad})}\right) ; 77.5,77.6(\mathrm{C}(4), \mathrm{C}(5)) ; 127.5,128.0,129.1,130.5,130.9131 .0(8$ arom. CH); 140.8, 157.2 (2 arom. C). Anal. calc. for $\mathrm{C}_{22} \mathrm{H}_{24} \mathrm{~S}_{2} \mathrm{Se}$ (431.52): C 61.24, H 5.61, S 14.86; found: C 61.17, H 5.39, S 15.16.

Spectroscopic data of $\mathbf{3 e}$, collected from the spectra of a mixture of $\mathbf{3 e}$ with the major product 4k: ${ }^{1} \mathrm{H}-\mathrm{NMR}\left(\mathrm{CDCl}_{3}\right)$ : 1.04-3.05 (m, $\left.14 \mathrm{H}\right)$; 3.78-3.96 $\left(m, \mathrm{CH}_{2}\right)$; $7.17-$ 7.96 ( $m, 8$ arom. H). ${ }^{13} \mathrm{C}-\mathrm{NMR}\left(\mathrm{CDCl}_{3}\right)$ : 26.3, 26.4, 41.9, $42.0\left(4 \mathrm{CH}_{(\mathrm{ad})}\right)$; 36.3, 36.6, 36.6, 37.0, $37.6\left(5 \mathrm{CH}_{2(\text { (ad) })}\right) ; 50.6\left(\mathrm{H}_{2} \mathrm{C}(5)\right) ; 72.5,78.5(\mathrm{C}(2), \mathrm{C}(4)) ; 127.1,127.4,127.8$, 127.9, 129.0, 130.7 (8 arom. CH); 144.2, 159.3 (2 arom. C). 
5.3. Reaction with (2f): 5,5-Bis(thiophen-2-yl)spiro[1,3-dithiolane-4,2'tricyclo[3.3.1.1 $1^{3,7}$ decane] (4I; major) and 4,4-Bis(thiophen-2-yl)spiro[1,3-dithiolane2,2'-tricyclo[3.3.1.1 ${ }^{3,7}$ ]decane] (3f; minor); crude product ratio: 82:18. Reaction time: 9 h. Isolated as a mixture of isomers. Yield $309 \mathrm{mg}(76 \%)$. Yellow crystals. M.p. 127.0$128.7^{\circ} . \mathrm{IR}(\mathrm{KBr}): 2899 s, 2851 s, 1628 w, 1442 m, 1425 m, 1225 m, 1097 m, 707 s, 697 s .{ }^{1} \mathrm{H}-$ $\operatorname{NMR}\left(\mathrm{CDCl}_{3}\right): 1.48-2.82(m, 28 \mathrm{H}) ; 3.78\left(s, \mathrm{CH}_{2}\right.$ of $\left.4 \mathbf{l}\right) ; 3.93\left(s, \mathrm{CH}_{2}\right.$ of 3f $) ; 7.00-7.41$ (m, 12 arom. H). ${ }^{13} \mathrm{C}-\mathrm{NMR}\left(\mathrm{CDCl}_{3}\right): 26.2,26.3,26.9,35.6,42.0\left(8 \mathrm{CH}_{(\mathrm{ad})}\right) ; 26.8$ $\left(\mathrm{H}_{2} \mathrm{C}(2)\right.$ of $\left.4 \mathbf{l}\right) ; 32.9,36.4,36.7,37.5,38.8,39.1\left(10 \mathrm{CH}_{2(\mathrm{ad})}\right) ; 52.4\left(\mathrm{H}_{2} \mathrm{C}(5)\right.$ of $\left.\mathbf{3 f}\right) ; 67.3$, 72.0, 76.5, $79.0(2 \mathrm{C}(4), 2 \mathrm{C}(5)) ; 125.1,125.1,125.9,126.6,127.7,127.0$ (12 arom. CH); 147.9 (2 arom. C of 4l); 150.0 (2 arom. C of 3f). ESI-MS (MeOH): 391 (100, $\left.[M+\mathrm{H}]^{+}\right)$

5.4. Reaction with 2g: 5,5-Bis(selenophen-2-yl)spiro[1,3-dithiolane-4,2'tricyclo[3.3.1.1 $\left.1^{3,7}\right]$ decane] (4m; major) and 4,4-Bis(selenophen-2-yl)spiro[1,3dithiolane-2,2'-tricyclo[3.3.1.1 $1^{3,7}$ decane] (3g; minor); crude product ratio: 74:26. Reaction time: 8 h. Isolated as a mixture of isomers. Yield: 325mg (64\%). M.p. 151.8153.0. IR (KBr): 2926s, 2899s, 2845s, 1625w, 1439m, 1234m, 1220m, 1097m, 717m, 695s, 703s. ${ }^{1} \mathrm{H}-\mathrm{NMR}\left(\mathrm{CDCl}_{3}\right): 1.39-2.76(m, 28 \mathrm{H}) ; 3.72\left(s, \mathrm{CH}_{2}\right.$ of $\left.4 \mathbf{m}\right) ; 3.82\left(s, \mathrm{CH}_{2}\right.$ of 3g); 7.07-7.81 (m, 12 arom. H). ${ }^{13} \mathrm{C}-\mathrm{NMR}\left(\mathrm{CDCl}_{3}\right)$ : 26.3, 26.4, 27.0, 27.1, 35.7, 41.9 (8 $\left.\mathrm{CH}_{(\mathrm{ad})}\right) ; 27.3\left(\mathrm{H}_{2} \mathrm{C}(2)\right.$ of $\left.4 \mathrm{~m}\right) ; 33.1,38.9,39.1,36.5,36.7,37.5\left(10 \mathrm{CH}_{2(\mathrm{ad})}\right) ; 53.4$ $\left(\mathrm{H}_{2} \mathrm{C}(5)\right.$ of 3g); 71.5, 79.3, 76.4, 76.4 (2 $\left.\mathrm{C}(4), 2 \mathrm{C}(5)\right) ; 127.7,129.3,131.0,129.2$, 130.1, 131.6 (12 arom. CH); 156.7 (2 arom. C of $\mathbf{3 g}), 158.0$ (2 arom. C of $\mathbf{4 m}$ ). Anal. calc. for $\mathrm{C}_{20} \mathrm{H}_{22} \mathrm{~S}_{2} \mathrm{Se}_{2}$ (484.45): C $49.59 \mathrm{H} 4.58 \mathrm{~S}$ 13.24; found: C $49.81 \mathrm{H} 4.45 \mathrm{~S} 13.66$.

5.5. Reaction with 2h: 5-(Furan-2-yl)-5-(thiophen-2-yl)spiro[1,3-dithiolane4,2'-tricyclo[3.3.1.1 $1^{3,7}$ decane] (4n; major) and 4-(Furan-2-yl)-4-(thiophen-2yl)spiro[1,3-dithiolane-2,2'-tricyclo[3.3.1.1 $\left.{ }^{3,7}\right]$ decane] (3h; minor); crude product ratio: 
77:23. Reaction time: 5 h. After CC, $\mathbf{4 n}$ and $\mathbf{3 h}$ were isolated as a mixture of regioisomers. Yield: $340 \mathrm{mg}(86 \%)$. After repeated crystallization from hexane, the major product $\mathbf{4 n}$ was isolated in pure form. Yellow crystals. M.p. 120.2-121.2 ${ }^{\circ}$. IR (KBr): 2899s, 2853s, 1636w, 1449m, 1235m, 1149m, 1097m, 1021m, 751m, 727s, 693s. ${ }^{1} \mathrm{H}-\mathrm{NMR}\left(\mathrm{CDCl}_{3}\right): 1.02-2.74(m, 14 \mathrm{H}) ; 3.73,3.84\left(A B, J_{\mathrm{AB}}=9.0, \mathrm{CH}_{2}\right) ; 6.33(d d, J=$ 3.0, 1.8, 1 arom. H); $6.75(d d, J=3.6,1.2,1$ arom. $\mathrm{H}) ; 6.99(d d, J=5.4,3.6,1$ arom. $\mathrm{H}) ; 7.20(d d, J=4.8,1.2,1$ arom. $\mathrm{H}) ; 7.43-7.44(m, 2$ arom. $\mathrm{H}) .{ }^{13} \mathrm{C}-\mathrm{NMR}\left(\mathrm{CDCl}_{3}\right)$ : $26.4\left(\mathrm{H}_{2} \mathrm{C}(2)\right) ; 26.9,27.2,35.0,35.7\left(4 \mathrm{CH}_{(\mathrm{ad})}\right) ; 32.6,33.1,38.3,39.2,40.0\left(5 \mathrm{CH}_{2(\mathrm{ad})}\right)$; 69.8, $77.1(\mathrm{C}(4), \mathrm{C}(5)) ; 111.0,112.3,124.8,126.6,127.5,141.4$ (6 arom. $\mathrm{CH})$; 146.5, 152.1 (2 arom. C). Anal. calc. for $\mathrm{C}_{20} \mathrm{H}_{22} \mathrm{OS}_{3}$ (374.59): C 64.13 H 5.92 S 25.68; found: C 64.05 H 6.00 S 25.65.

Spectroscopic data of $\mathbf{3 h}$, collected from the spectra from a mixture of $\mathbf{3 h}$ with the major product $4 \mathbf{n} .{ }^{1} \mathrm{H}-\mathrm{NMR}\left(\mathrm{CDCl}_{3}\right)$ : $1.02-2.74(m, 14 \mathrm{H}) ; 3.75-4.03\left(m, \mathrm{CH}_{2}\right)$; 6.32-7.38 (m, 6 arom. H). ${ }^{13} \mathrm{C}-\mathrm{NMR}\left(\mathrm{CDCl}_{3}\right): 26.2,26.4,42.0,42.1 \quad\left(4 \mathrm{CH}_{(\mathrm{ad})}\right) ; 36.3$, 36.5, 36.7, 36.9, $37.6\left(5 \mathrm{CH}_{2(\mathrm{ad})}\right) ; 48.8\left(\mathrm{H}_{2} \mathrm{C}(5)\right)$; 65.5, $78.8(\mathrm{C}(2), \mathrm{C}(4))$; 109.0, 110.3, 125.1, 125.7, 126.6, 142.1 (6 arom. CH); 147.6, 155.8 (2 arom. C).

6. Reactions of Hetaryl Phenyl Thioketones $\mathbf{2 d - 2 e}$ or Bishetaryl Thioketones $\mathbf{2 f -}$ 2h with 1,1,3,3-Tetramethyl-5-thia-7,8-diazaspiro[3.4]oct-7-en-2-one (5d). - General Procedure. A soln. of $\mathbf{5 d}(1.1 \mathrm{mmol})$ and a thioketones $\mathbf{2 d}-\mathbf{2 h}(1.05 \mathrm{mmol})$ in freshly distilled THF $(2.5 \mathrm{ml})$ was heated in an oil bath $\left(45-50^{\circ}\right)$ until the color of the thioketone disappeared; a gas burette indicated the liberation of a stoichiometric amount of $\mathrm{N}_{2}$. After removal of the solvent under vacuum, the residue was subjected to ${ }^{1} \mathrm{H}$ NMR analysis in $\mathrm{CDCl}_{3}$ with a weighed amount of 1,1,2,2-tetrachloroethane as a standard. 
6.1. Reaction with 2d: 1,1,3,3-Tetramethyl-8-phenyl-8-(thiophen-2-yl)-5,7dithiaspiro[3.4]octan-2-one (4o; major) and 1,1,3,3-Tetramethyl-6-phenyl-6-(thiophen2-yl)-5,8-dithiaspiro[3.4]octan-2-one (3i; minor); crude product ratio: 86:14. Reaction time: $3.5 \mathrm{~h}$. The crude mixture was purified by $\mathrm{CC}\left(\mathrm{CH}_{2} \mathrm{Cl}_{2} /\right.$ hexane $\left.4: 6\right)$. Product isolated as a 86:14 mixture of regioisomers. Yield: $372 \mathrm{mg}(94 \%)$. Colorless crystals. M.p. 109.0-112.0 ${ }^{\circ}$ IR (KBr): $1778 s(\mathrm{C}=\mathrm{O}), 1636 w, 1597 w, 1443 m, 1230 m, 1164 m$, 1022m, 744m, 707s. ${ }^{1} \mathrm{H}-\mathrm{NMR}\left(\mathrm{CDCl}_{3}\right): 1.26,1.33,1.36,1.38,1.47,1.58,1.65,1.72(8 s$, $8 \mathrm{Me}) ; 3.62-3.66\left(m, \mathrm{CH}_{2}\right.$ of $\left.4 \mathbf{4}\right) ; 3.83,3.92\left(A B, J_{\mathrm{AB}}=12.0, \mathrm{CH}_{2}\right.$ of $\left.\mathbf{3 i}\right) ; 6.86-7.82(m$, 16 arom. H). ${ }^{13} \mathrm{C}-\mathrm{NMR}\left(\mathrm{CDCl}_{3}\right): 22.3,22.5,24.6,24.8,24.8,25.5,25.68,25.70(8 \mathrm{Me})$; $26.8\left(\mathrm{H}_{2} \mathrm{C}(6)\right.$ of $\left.4 \mathbf{0}\right) ; 51.7\left(\mathrm{H}_{2} \mathrm{C}(7)\right.$ of $\left.3 \mathbf{i}\right) ; 66.5$ (br.), 66.6, 67.0, 68.8 (br.) (2 C(1), 2 $\mathrm{C}(3)) ; 71.0,74.5,75.8,76.1(\mathrm{C}(4), \mathrm{C}(8)$ of $4 \mathbf{0}, \mathrm{C}(4), \mathrm{C}(7)$ of 3i); 125.2, 126.1, 126.3, 126.6, 127.4, 127.6, 127.7, 127.9, 128.1, 128.4, 129.1, 130.2 (16 arom. CH); 132.2, 134.1 (2 arom. $\mathrm{C}$ of 3i); 143.4, 150.3 (2 arom. $\mathrm{C}$ of 4o); $219.9(\mathrm{C}=\mathrm{O}$ of 4o); $220.1(\mathrm{C}=\mathrm{O}$

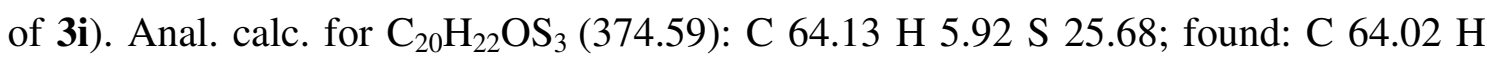
$5.93 \mathrm{~S} 25.95$.

6.2. Reaction with 2e: 1,1,3,3-Tetramethyl-8-phenyl-8-(selenophen-2-yl)-5,7dithiaspiro[3.4]octan-2-one (4p; major) and 1,1,3,3-Tetramethyl-6-phenyl-6(selenophen-2-yl)-5,8-dithiospiro[3.4]octan-2-one (3k; minor); crude product ratio: 96:4. Reaction time: $6.5 \mathrm{~h}$. The crude product was crystallized from $\mathrm{CH}_{2} \mathrm{Cl}_{2} / \mathrm{MeOH}$. Yield: $420 \mathrm{mg}(95 \%)$. Colorless crystals. M.p. 140.0-142.0. IR (KBr): 1170s (C=O), 1443m, 1227m, 1164w, 1020w, 706s, 695s. ${ }^{1} \mathrm{H}-\mathrm{NMR}\left(\mathrm{CDCl}_{3}\right):$ 1.23, 1.25, 1.28, 1.31, $1.47,1.61,1.62,1.71(8 s, 8 \mathrm{Me}) ; 3.70-3.74\left(m, \mathrm{CH}_{2}\right.$ of $\left.4 \mathbf{p}\right) ; 3.78-3.92\left(m, \mathrm{CH}_{2}\right.$ of $\left.3 \mathbf{k}\right)$; 7.13-7.97 (m, 16 arom. H). ${ }^{13} \mathrm{C}-\mathrm{NMR}\left(\mathrm{CDCl}_{3}\right): 22.1,22.5,24.5,24.8,25.2,25.5,25.7$, $25.8(8 \mathrm{Me}) ; 27.0\left(\mathrm{H}_{2} \mathrm{C}(6)\right.$ of $\left.\mathbf{4 p}\right) ; 52.1\left(\mathrm{H}_{2} \mathrm{C}(7)\right.$ of $\left.\mathbf{3 k}\right) ; 60.2,65.4,66.2,69.7$ (2 C(1), 2 $\mathrm{C}(3)) ; 67.1,73.0,76.1,76.7(\mathrm{C}(4), \mathrm{C}(8)$ of $\mathbf{4 p}, \mathrm{C}(4), \mathrm{C}(7)$ of $\mathbf{3 k}) ; 127.4,127.5,127.8$, 
128.0, 128.3, 128.4 (br.), 128.9, 129.1, 130.8 (br.), 130.9, 131.3, 131.7 (16 arom. CH); 140.8, 160.8, 143.5, 158.1 (4 arom. C); 219.7, 219.9 (2 $\mathrm{C}=\mathrm{O})$. Anal. calc. for $\mathrm{C}_{20} \mathrm{H}_{22} \mathrm{OS}_{2} \mathrm{Se}$ (421.49): C 56.99 H 5.26 S 15.21; found: C 56.55 H 5.12 S 15.17.

6.3. Reaction with $\mathbf{2 h}$ : 1,1,3,3-Tetramethyl-8-(furan-2-yl)-8-(thiophen-2-yl)-5,7dithiaspiro[3.4]octan-2-one (4q; major) and 1,1,3,3-Tetramethyl-6-(furan-2-yl)-6(thiophen-2-yl)-5,8-dithiospiro[3.4]octan-2-one (31; minor); crude product ratio: 91:9. Reaction time: $4 \mathrm{~h}$. The crude product was purified by $\mathrm{CC}\left(\mathrm{CH}_{2} \mathrm{Cl}_{2} /\right.$ hexane $\left.4: 6\right)$. Yield: $370 \mathrm{mg}(96 \%)$. Yellow crystals. M.p. 130.0-132.4 $. \mathrm{IR}(\mathrm{KBr}): 1784 \mathrm{~s}(\mathrm{C}=\mathrm{O}), 1464 m$, $1381 s, 1227 s, 1130 m, 1074 m, 1027 s, 1016 s, 808 s, 742 s, 695 s, 593 m .{ }^{1} \mathrm{H}-\mathrm{NMR}\left(\mathrm{CDCl}_{3}\right)$ : $0.81,1.24,1.29,1.34,1.38,1.45,1.57,1.59(8 s, 8 \mathrm{Me}) ; 3.61-3.92\left(m, \mathrm{CH}_{2}\right.$ of 3l); 3.86$3.90\left(m, \mathrm{CH}_{2}\right.$ of $\left.\mathbf{4 q}\right) ; 6.35-7.44\left(m, 12\right.$ arom. H). ${ }^{13} \mathrm{C}-\mathrm{NMR}\left(\mathrm{CDCl}_{3}\right): 19.3,22.1,22.4$, 23.3, 24.6, 24.7 28.3, $28.9(8 \mathrm{Me}) ; 29.1\left(\mathrm{H}_{2} \mathrm{C}(6)\right.$ of $\left.\mathbf{4 q}\right)$; $50.6\left(\mathrm{H}_{2} \mathrm{C}(7)\right.$ of $\left.\mathbf{3 1}\right)$; 65.6, 66.6, 67.0, 67.3 (2 C(1), $2 \mathrm{C}(3))$; 65.0, 67.2, 76.8, $77.3(\mathrm{C}(4), \mathrm{C}(8)$ of $\mathbf{4 q}, \mathrm{C}(4), \mathrm{C}(7)$ of 31); $109.1,110.4,111.6,112.0,125.3,125.7,126.0,126.2,126.4,126.8,140.8,142.4(12$ arom. $\mathrm{CH}$ ); 146.8, 148.1, 155.0, 152.2 (4 arom. C); 219.4, 219.7 (2 C=O). Anal. calc. for $\mathrm{C}_{18} \mathrm{H}_{20} \mathrm{O}_{2} \mathrm{~S}_{3}$ (364.55): C 59.31 H 5.53 S 26.39; found: C 58.93 H 5.19 S 26.04.

6.4. Reaction with 2f: 1,1,3,3-Tetramethyl-8,8-bis(thiophen-2-yl)-5,7dithiaspiro[3.4]octan-2-one (4r). The reaction was complete after $2 \mathrm{~h}$. The solvent was evaporated, and the crude product was purified by $\mathrm{CC}\left(\mathrm{CH}_{2} \mathrm{Cl}_{2} /\right.$ hexane 4:6). Yield: 320 mg (81\%). Colorless crystals. M.p. 140.6-141.5 . IR (KBr): 1774s (C=O), 1630w, 1385w, 1234m, 710s. ${ }^{1} \mathrm{H}-\mathrm{NMR}\left(\mathrm{CDCl}_{3}\right): 1.45,1.64(2 s, 4 \mathrm{Me}) ; 3.80\left(s, \mathrm{CH}_{2},\right) ; 6.94$ 6.95 (m, 2 arom. H); 7.24-7.25 (m, 2 arom. H); 7.29-7.30 (m, 2 arom. H). ${ }^{13} \mathrm{C}-\mathrm{NMR}$ $\left(\mathrm{CDCl}_{3}\right)$ : 24.1, 26.5 (4 Me); $28.3\left(\mathrm{H}_{2} \mathrm{C}(6)\right) ; 67.0(\mathrm{C}(1), \mathrm{C}(3)) ; 70.1,76.6(\mathrm{C}(4), \mathrm{C}(8))$; 125.7, 126.3, 127.8 (6 arom. $\mathrm{CH}) ; 148.2$ (2 arom. C); $219.5(\mathrm{C}=\mathrm{O})$. Anal. calc. for $\mathrm{C}_{18} \mathrm{H}_{20} \mathrm{OS}_{4}$ (380.62): C 56.80 H 5.30 S 33.70; found: C 57.02 H 5.52 S 33.68. 
Suitable crystals for the X-ray crystal-structure determination were grown from $\mathrm{MeOH} / \mathrm{CH}_{2} \mathrm{Cl}_{2}$ in the refrigerator.

6.5. Reaction with 2g: 1,1,3,3-Tetramethyl-8,8-bis(selenophen-2-yl)-5,7dithiaspiro[3.4]octan-2-one (4s). The reaction was complete after $4 \mathrm{~h}$. The crude product was crystallized from $\mathrm{MeOH}$. Yield: $323 \mathrm{mg}$ (85\%). Pale yellow crystals. M.p. 157.0-158.0 $(\mathrm{MeOH})$. IR $(\mathrm{KBr}): 1770 s(\mathrm{C}=\mathrm{O}), 1442 m, 1383 m, 1232 s, 1012 m, 808 m$, 696s. ${ }^{1} \mathrm{H}-\mathrm{NMR}\left(\mathrm{CDCl}_{3}\right): 1.45,1.63(2 s, 4 \mathrm{Me}) ; 3.86\left(s, \mathrm{CH}_{2}\right) ; 7.17-7.18(m, 2$ arom. $\mathrm{H})$; 7.37-7.38 (m, 2 arom. H); 7.95-7.96 (m, 2 arom. H). ${ }^{13} \mathrm{C}-\mathrm{NMR}\left(\mathrm{CDCl}_{3}\right): 24.4,26.8$ (4 Me); $28.9\left(\mathrm{H}_{2} \mathrm{C}(6)\right) ; 66.9(\mathrm{C}(1), \mathrm{C}(3)) ; 74.2,76.5(\mathrm{C}(4), \mathrm{C}(8)) ; 129.0,129.2,132.4(6$ arom. $\mathrm{CH}) ; 156.6$ (2 arom. C); $219.4(\mathrm{C}=\mathrm{O})$. Anal. calc. for $\mathrm{C}_{18} \mathrm{H}_{20} \mathrm{OS}_{2} \mathrm{Se}_{2}$ (474.41): C 45.57 H 4.25 S 13.52; found: C 45.84 H 4.32 S 13.55.

7. Competition Experiments. - a) Bis(thiophen-2-yl)methanethione (2f) versus 9H- Fluorene-9-thione (2c) with 2,5-Dihydro-2,2-diphenyl-1,3,4-thiadiazole (5b). A soln. of thiobenzophenone (2b, $1 \mathrm{mmol})$ in $2 \mathrm{ml}$ of THF was cooled to $-70^{\circ}$ and treated with small portions of a soln. of $\mathrm{CH}_{2} \mathrm{~N}_{2}$ in $\mathrm{Et}_{2} \mathrm{O}$, until the dark-blue color disappeared. Then, equimolar amounts of $\mathbf{2 f}(1 \mathrm{mmol})$ and $\mathbf{2 c}(1 \mathrm{mmol})$ dissolved in $3 \mathrm{ml}$ of THF were added at $-70^{\circ}$ and the mixture was kept at $-45^{\circ}$ to $-40^{\circ}$ (acetone/dry ice bath) for 2 h. After that time the soln. was allowed to warm slowly to r.t. and kept at r.t. for $c a$. 30 min, and then the solvent was evaporated. The residue was subjected to ${ }^{1} \mathrm{H}-\mathrm{NMR}$ analysis in $\mathrm{CDCl}_{3}$ with a weighed amount of 1,1,2,2-tetrachloroethane as standard. The only observed product was the known 5,5'-diphenylspiro[9H-fluorene-9,4'[1,3]dithiolane] [3b]. Yield: $99 \%\left({ }^{1} \mathrm{H}\right.$ NMR).

b) Bis(thiophen-2-yl)methanethione (2f) versus Thiobenzophenone (2b) with $\mathbf{5 b}$. In analogy to the procedure described above, a soln. of $\mathbf{2 b}(0.57 \mathrm{mmol})$ in $2 \mathrm{ml}$ of THF at $-70^{\circ}$ was treated with an ethereal $\mathrm{CH}_{2} \mathrm{~N}_{2}$ soln., followed by a soln. of $\mathbf{2 f}(0.57 \mathrm{mmol})$ 
and $2 \mathrm{c}(0.57 \mathrm{mmol})$ in $3 \mathrm{ml}$ of THF. The mixture was stirred at $-45^{\circ}$ to $-40^{\circ}$ for $2 \mathrm{~h}$ and then allowed to warm slowly to r.t. After $30 \mathrm{~min}$. at r.t., the solvent was evaporated and the residue was subjected to ${ }^{1} \mathrm{H}-\mathrm{NMR}$ analysis $\left(\mathrm{CDCl}_{3}, 1,1,2,2\right.$-tetrachloroethane as standard). The crude product was identified as a mixture of the known 4,4,5,5tetraphenyl-1,3-dithiolane [3b] (40\%) and 5,5-diphenyl-4,4-bis(thiophen-2-yl)-1,3dithiolane (4f) $\left(29 \%,{ }^{1} \mathrm{H}\right.$ NMR).

8. X-Ray Crystal Structure Determination of $\left.\mathbf{4 r}(\text { Table and Figure })^{2}\right)$. All measurements were made on an Agilent Technologies SuperNova area-detector diffractometer [14] using MoK $\alpha$ radiation $(\lambda=0.71073 \AA$ ) from a micro-focus X-ray source and an Oxford Instruments Cryojet XL cooler. Data reduction was performed with CrysAlisPro [14]. The intensities were corrected for Lorentz and polarization effects, and an empirical absorption correction using spherical harmonics [14] was applied. Equivalent reflections, other than Friedel pairs, were merged. The data collection and refinement parameters are given in the Table. A view of the molecule is shown in the Figure. The structure was solved by direct methods using SHELXS-2013 [15], which revealed the positions of all non-H-atoms. There are two symmetryindependent molecules in the asymmetric unit. The atomic coordinates of the two molecules were tested carefully for a relationship from a higher symmetry space group using the program PLATON [16], but none could be found. Both molecules show disorder of both thiophene rings due to $180^{\circ}$ rotation of each ring around its parent $\mathrm{C}-\mathrm{C}$ bond which swaps the positions of the $\mathrm{S}$ - and $\mathrm{C}$-atoms in the 2,5-positions of the ring.

2) CCDC-1052007 contains the supplementary crystallographic data for this paper. These data can be obtained free of charge from the Cambridge Crystallographic Data Centre, via www.ccdc.cam.ac.uk/data_request/cif. 
Two positions were defined for these S- and C-atoms in each disordered ring and the site occupation factors of the major orientations of these rings refined to 0.680(4) and $0.845(3)$ in molecule A and 0.954(3) and 0.794(3) in molecule B. Similarity restraints were applied to the chemically equivalent bond lengths involving all disordered $\mathrm{S}$ - and $\mathrm{C}$-atoms. In addition, the bond lengths involving disordered $\mathrm{C}$-atoms were restrained to 1.40(1) $\AA$. Neighboring disordered atoms between each orientation of the disordered thiophene rings were restrained to have similar atomic displacement parameters. One thiophene ring was additionally restrained to be planar. The non-H-atoms were refined anisotropically. All of the $\mathrm{H}$-atoms were placed in geometrically calculated positions and refined by using a riding model where each $\mathrm{H}$-atom was assigned a fixed isotropic displacement parameter with a value equal to $1.2 \mathrm{U}_{\mathrm{eq}}$ of its parent $\mathrm{C}$-atom $\left(1.5 \mathrm{U}_{\mathrm{eq}}\right.$ for the Me groups). The refinement of the structure was carried out on $F^{2}$ by using fullmatrix least-squares procedures, which minimized the function $\Sigma w\left(F_{\mathrm{o}}{ }^{2}-F_{\mathrm{c}}{ }^{2}\right)^{2}$. A correction for secondary extinction was not applied. Refinement of the absolute structure parameter [17] yielded a value of $0.01(1)$, which confidently confirms that the refined model represents the true absolute structure. Neutral atom scattering factors for non-H-atoms were taken from [18a], and the scattering factors for $\mathrm{H}$-atoms were taken from [19]. Anomalous dispersion effects were included in $F_{\mathrm{c}}$ [20]; the values for $f^{\prime}$ and $f^{\prime \prime}$ were those of [18b]. The values of the mass attenuation coefficients are those of [18c]. The SHELXL-2014 program [21] was used for all calculations.

Table. Crystallographic Data for Compound $\mathbf{4 r}$ 
[1] a) G. Mlostoń, H. Heimgartner, in 'Synthetic Applications of 1,3-Dipolar Cycloaddition Chemistry Toward Heterocycles and Natural Products', Eds. A. Padwa, W. H. Pearson, J. Wiley \& Sons, New York, 2002, p. 315; b) G. Mlostoń, H. Heimgartner, Curr. Org. Chem. 2011, 15, 675.

[2] R. Huisgen, X. Li, H. Giera, E. Langhals, Helv. Chim. Acta 2001, 84, 981.

[3] a) R. Huisgen, I. Kalvinsch, X. Li, G. Mlostoń, Eur J. Org. Chem. 2000, 1685; b) R. Huisgen, X. Li, G. Mlostoń, C. Fulka, Eur. J. Org. Chem. 2000, 1695.

[4] G. Mlostoń, R. Huisgen, Heterocycles 1985, 23, 2201.

[5] G. Mlostoń, K. Urbaniak, K. Gebicki, P. Grzelak, H. Heimgartner, Heteroatom Chem. 2014, 25, 584.

[6] G. Mlostoń, K. Urbaniak, A. Linden, H. Heimgartner, Helv. Chim. Acta. 2015, 98, submitted.

[7] a) R. Sustmann, W. Sicking, R. Huisgen, Chem. Eur. J. 2003, 9, 2245; b) R. Huisgen, G. Mloston, K. Polborn, R. Sustmann, Chem. Eur. J. 2003, 9, 2256.

[8] G. Mloston, H. Heimgartner, Pol. J. Chem.. 2000, 74, 1503.

[9] C.K. Johnson, ORTEPII, Report ORNL-5138, Oak Ridge National Laboratory, Oak Ridge, Tennessee, 1976.

[10] W. Freund, S. Huenig, J. Org. Chem. 1987, 52, 2154; W. Freund, S. Huenig, Helv. Chim. Acta 1987, 70, 929.

[11] a) R. A. Firestone, J. Org. Chem. 1968, 33, 2285; b) R. A. Firestone, Tetrahedron 1977, 33, 3009.

[12] R. Huisgen, G. Mloston, C. Fulka, Heterocycles 1985, 23, 2207.

[13] R. Huisgen, G. Mlostoń, Tetrahedron Lett. 1985, 26, 1049.

[14] CrysAlisPro, Version 1.171.37.31d, Agilent Technologies, Yarnton, Oxfordshire, England, 2014. 
[15] G. M. Sheldrick, Acta Crystallogr. Sect. A 2008, 64, 112.

[16] A. L. Spek, Acta Crystallogr. Sect. C 2015, 71, 9.

[17] a) H.D. Flack, G. Bernardinelli, Acta Crystallogr., Sect. A, 1999, 55, 908-915; b) H.D. Flack, G. Bernardinelli, J. Appl. Crystallogr., 2000, 33, 1143-1148.

[18] a) E. N. Maslen, A. G. Fox, M. A. O'Keefe, in 'International Tables for Crystallography', Ed. A. J. C. Wilson, Kluwer Academic Publishers, Dordrecht, 1992, Vol. C, Table 6.1.1.1, pp. 477-486; b) D. C. Creagh, W. J. McAuley, in 'International Tables for Crystallography', Ed. A. J. C. Wilson, Kluwer Academic Publishers, Dordrecht, 1992, Vol. C, Table 4.2.6.8, pp. 219-222; c) D. C. Creagh, J. H. Hubbell, in 'International Tables for Crystallography', Ed. A. J. C. Wilson, Kluwer Academic Publishers, Dordrecht, 1992, Vol. C, Table 4.2.4.3, pp. 200206.

[19] R. F. Stewart, E. R. Davidson, W. T. Simpson, J. Chem. Phys. 1965, 42, 3175.

[20] J. A. Ibers, W. C. Hamilton, Acta Crystallogr. 1964, 17, 781.

[21] G. M. Sheldrick, Acta Crystallogr., Sect. C 2015, 71, 3. 
Table. Crystallographic Data for Compound $\mathbf{4 r}$

Crystallized from

Empirical formula

Formula weight $\left[\mathrm{g} \mathrm{mol}^{-1}\right.$ ]

Crystal color, habit

Crystal dimensions [mm]

Temperature $[\mathrm{K}]$

Crystal system

Space group

Z

Reflections for cell determination

$2 \theta$ range for cell determination $\left[{ }^{\circ}\right]$

Unit cell parameters $a[\AA]$ $b[\AA]$

$c[\AA]$

$\beta\left[^{\circ}\right]$

$V\left[\AA^{3}\right]$
$\mathrm{MeOH}$

$\mathrm{C}_{18} \mathrm{H}_{20} \mathrm{OS}_{4}$

380.60

colorless, prism

$0.23 \times 0.24 \times 0.37$

160(1)

monoclinic

$P 2_{1}$

4

14706

5-60

$8.69316(11)$

12.82765(15)

15.94089(19)

90.7785(11)

1777.45(4)

1.422

0.535

$\omega$

60.9

$0.932 ; 1.000$

23111

9322

8753

9322

499; 319

0.0301

0.0708

Weights: $w=\left[\sigma^{2}\left(F_{\mathrm{O}}{ }^{2}\right)+(0.0314 P)^{2}+0.4989\right]^{-1}$ where $P=\left(F_{\mathrm{O}}^{2}+2 F_{\mathrm{c}^{2}}^{2}\right) / 3$

Goodness of fit

1.039

Final $\Delta_{\max } / \sigma$

0.001

$\Delta \rho$ (max; min) [e $\AA^{-3}$ ]

$0.55 ;-0.43$ 
Legends

Figure. ORTEP plot [9] of the molecular structure of one of the two symmetryindependent molecules of $\mathbf{4 r}$ (with $50 \%$ probability ellipsoids; arbitrary numbering of the atoms; only the major conformations of the disordered thiophene rings are shown).

Table. Crystallographic Data for Compound $\mathbf{4 r}$ 
Scheme 1

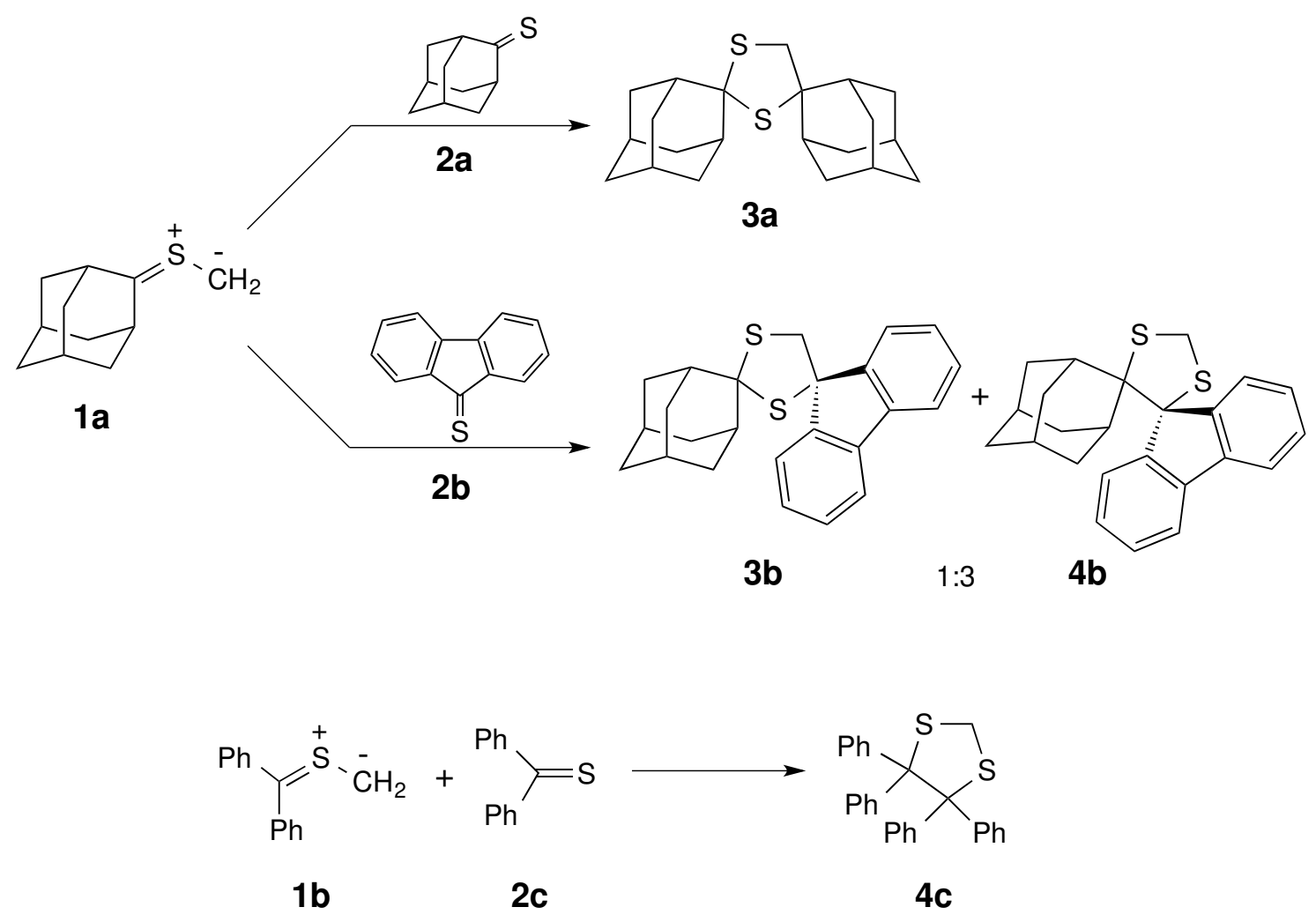


Scheme 2

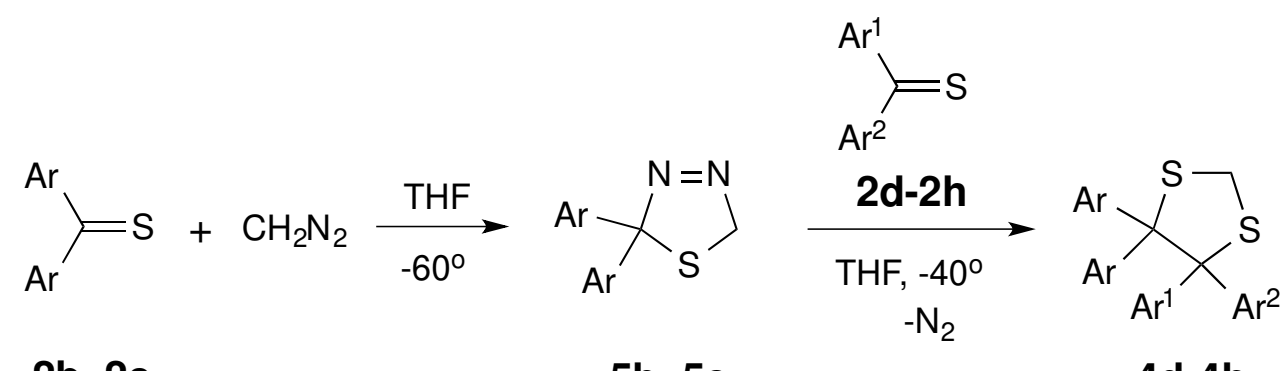

$2 b, 2 c$

$5 b, 5 c$

4d-4h

$\mathbf{b}: \mathrm{Ar}=\mathrm{Ph} ; \mathbf{c}:{ }_{\mathrm{Ar}}^{\mathrm{Ar}}>$

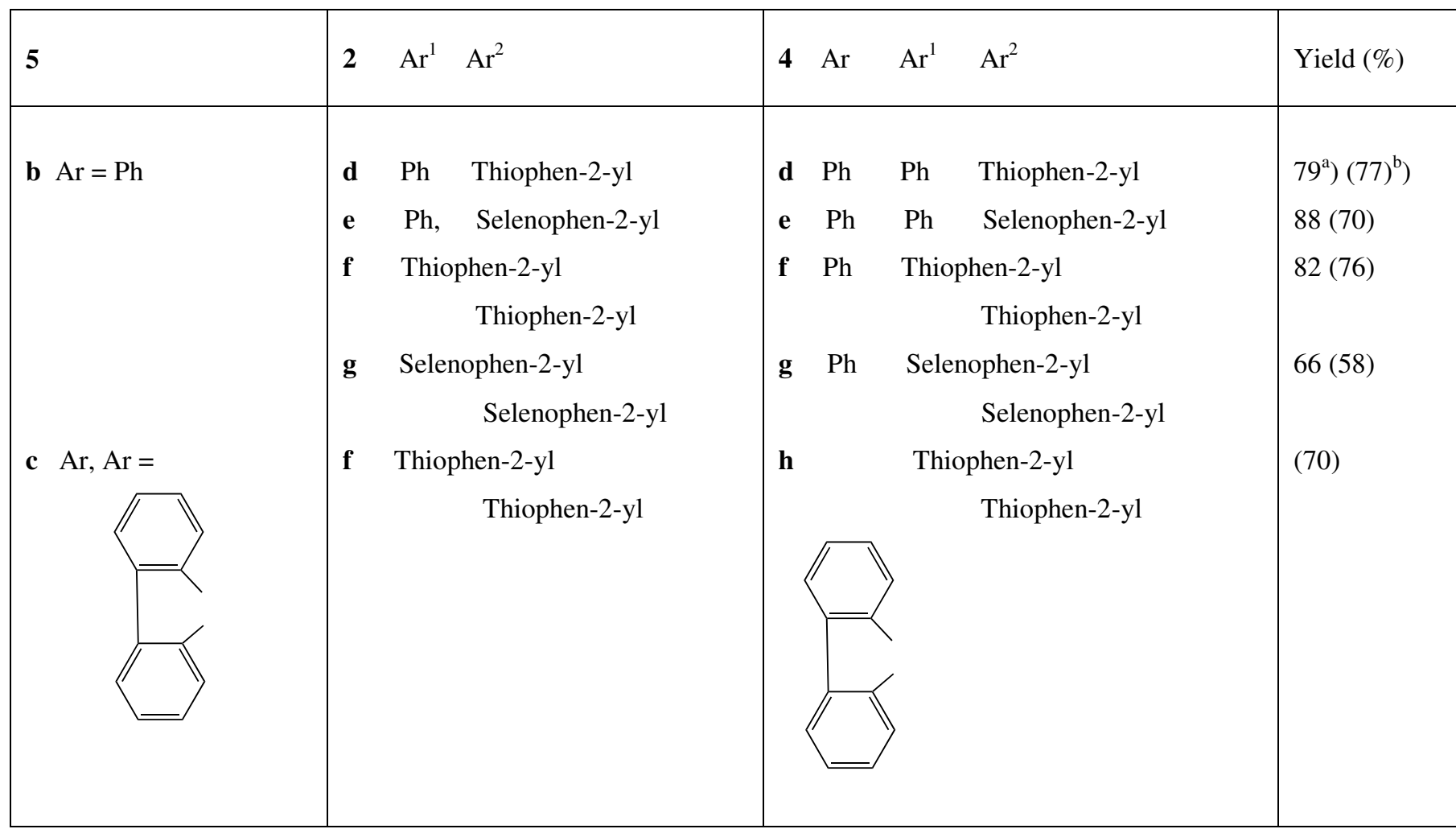

a) Yields determined by ${ }^{1} \mathrm{H}-\mathrm{NMR}$ with a weighed amount of 1,1,2,2-tetrachloroethane as a standard

b) Yields of isolated product 
Scheme 3

${ }_{N}^{S}=N+2 \mathbf{d}-\mathbf{2 h} \frac{\mathrm{THF}, 45^{\circ}}{-\mathrm{N}_{2}}$

$5 a$<smiles>[R17]C1([Al])CSC2(S1)C1CC3CC(C1)C1(SCSC1([Al])[Te])C2C3</smiles>

$3 d-3 h$

$4 i-4 n$

\begin{tabular}{|c|c|c|c|c|c|}
\hline \multirow[t]{2}{*}{2} & \multirow[t]{2}{*}{$\mathrm{Ar}^{1}$} & \multirow[t]{2}{*}{$\mathrm{Ar}^{2}$} & \multicolumn{2}{|c|}{ Yield $\left.(\%)^{\mathrm{a}}\right)$} & \multirow[t]{2}{*}{ Yield $\left.(\%)^{\mathrm{b}}\right)$} \\
\hline & & & 3 & 4 & \\
\hline d & $\mathrm{Ph}$ & Thiophen-2-yl & d 23 & i $\quad 70$ & 82 \\
\hline e & $\mathrm{Ph}$ & Selenophen-2-yl & e $\quad 21$ & k 71 & 80 \\
\hline f & Thiophen-2-yl & Thiophen-2-yl & f $\quad 16$ & l 75 & 76 \\
\hline g & Selenophen-2-yl & Selenophen-2-yl & g $\quad 22$ & m 63 & 64 \\
\hline h & Thiophen-2-yl & Furan-2-yl & h $\quad 21$ & n 70 & 86 \\
\hline
\end{tabular}

a) Yields determined by ${ }^{1} \mathrm{H}-\mathrm{NMR}$ with a weighed amount of 1,1,2,2-tetrachloroethane as a standard

b) Yields of isolated products (as mixtures of $\mathbf{3}$ and $\mathbf{4}$ ) 


\section{Scheme 4}

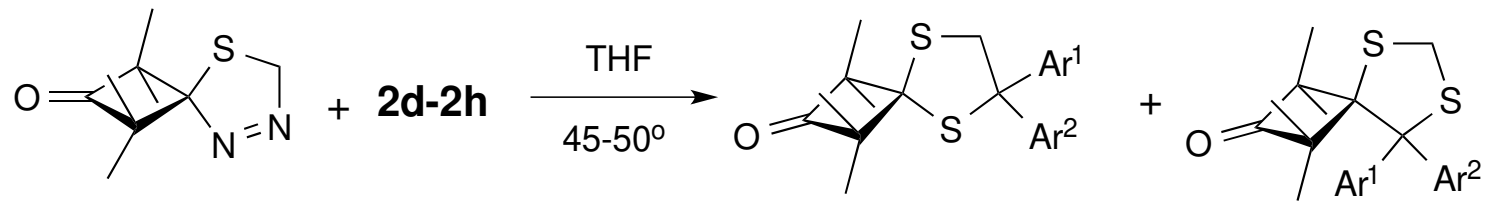

$5 d$

3i-e

40-s

\begin{tabular}{|c|c|c|c|c|c|}
\hline \multirow[t]{2}{*}{2} & \multirow[t]{2}{*}{$\mathrm{Ar}^{1}$} & \multirow[t]{2}{*}{$A r^{2}$} & \multicolumn{2}{|c|}{ Yield $\left.(\%)^{\mathrm{a}}\right)$} & \multirow[t]{2}{*}{ Yield $\left.(\%)^{\mathrm{b}}\right)$} \\
\hline & & & 3 & 4 & \\
\hline d & $\mathrm{Ph}$ & Thiophen-2-yl & i 13 & о 84 & 94 \\
\hline $\mathbf{e}$ & $\mathrm{Ph}$ & Selenophen-2-yl & $\mathbf{k}$ & $\mathbf{p} \quad 91$ & 95 \\
\hline $\mathbf{h}$ & Thiophen-2-yl & Furan-2-yl & 1 & $\mathbf{q} \quad 91$ & 96 \\
\hline $\mathbf{f}$ & Thiophen-2-yl & Thiophen-2-yl & - & r 83 & 81 \\
\hline $\mathbf{g}$ & Selenophen-2-yl & Selenophen-2-yl & - & s $\quad 95$ & 85 \\
\hline
\end{tabular}

a) Yields determined by ${ }^{1} \mathrm{H}-\mathrm{NMR}$ with a weighed amount of 1,1,2,2-tetrachloroethane as a standard

b) Yields of isolated products (as mixtures of 3 and $\mathbf{4}$ or as pure compounds $\mathbf{4 r}$ and $\mathbf{4 s}$ ) 
Formulae

$\left\langle\mathrm{s}={ }_{\mathrm{Ar}}^{\mathrm{Ar}}\right.$

$6 a$

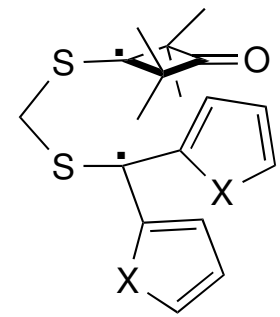

6b $X=S e, S$

Figure

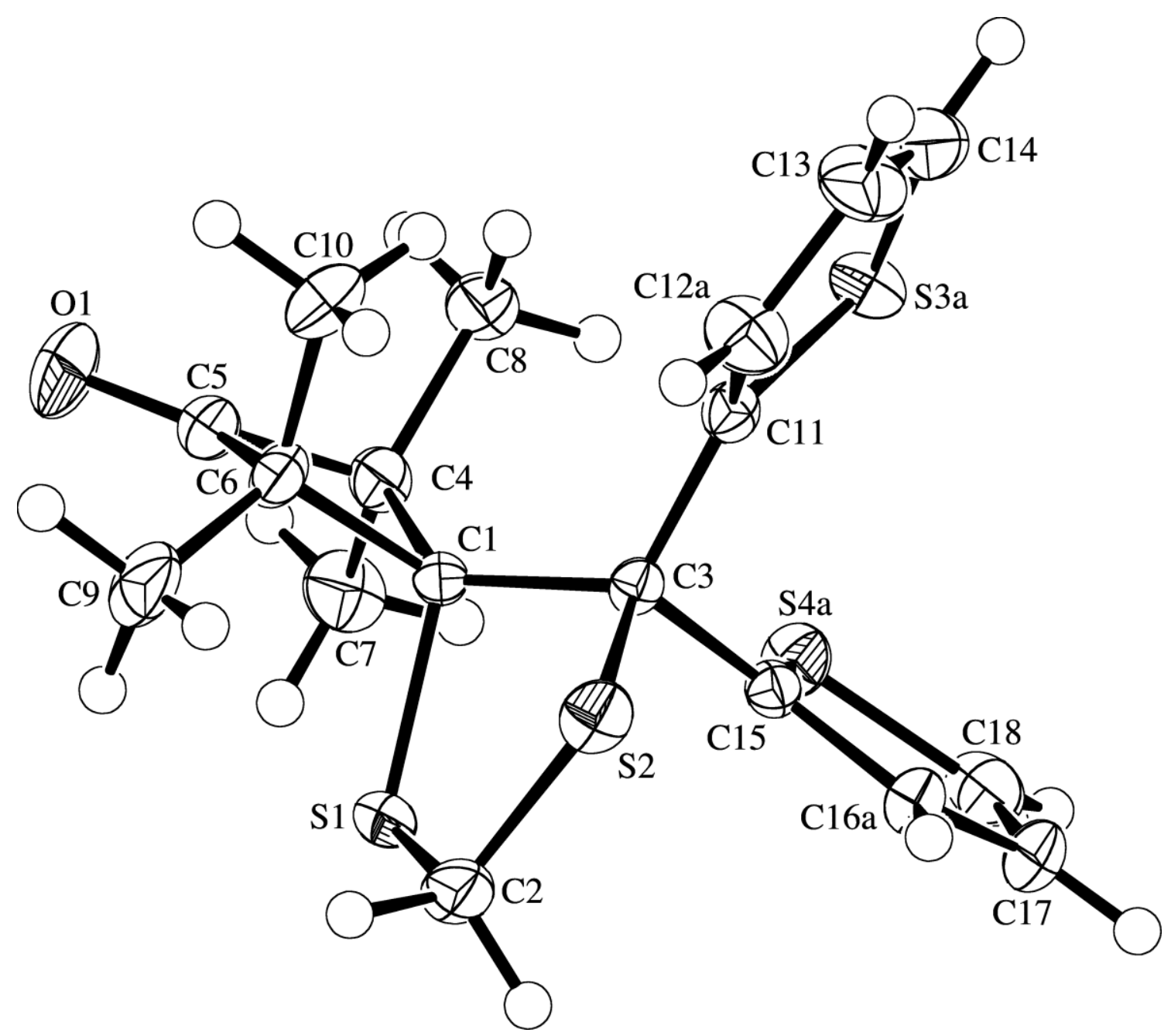




\section{Graphical Abstract}

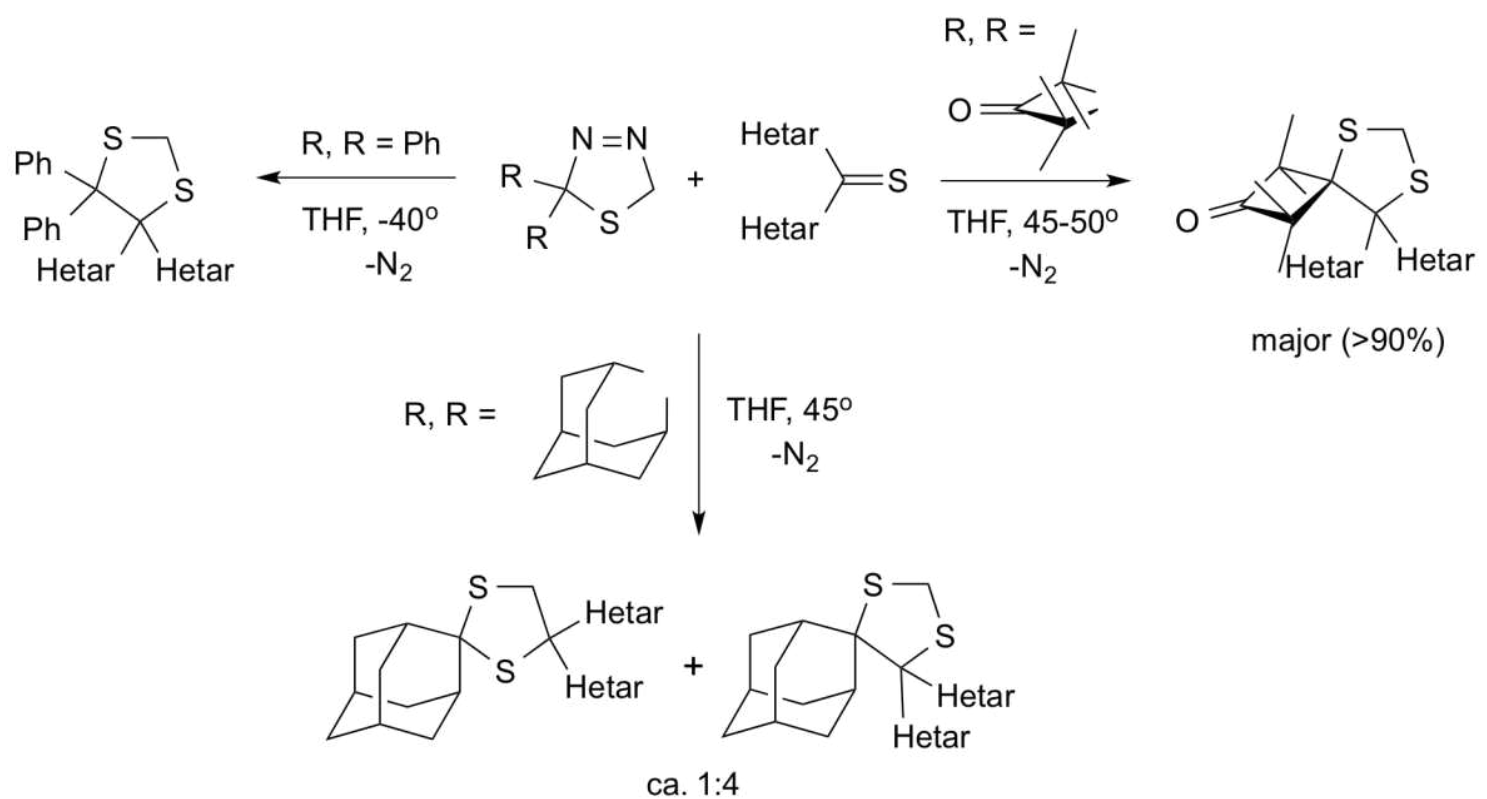

